\title{
A case study on student perception of online lecturing
}

\section{Domenik Wolff-Boenisch}

School of Earth and Planetary Sciences, Curtin University, GPO Box U1987, Perth, WA6845, Australia.

\begin{abstract}
This case study looks at implications of transitioning live to recorded lectures, a subject that has acquired an acute importance given COVID19 and the unexpected need to move lectures online. Over a period of six years, from 2015 to 2020, a questionnaire was handed out at the end of a 'unit' on environmental geoscience; a 'unit' at Australian universities represents a 'course' in the European and American tertiary system. This is a 2nd semester, 3rd year core unit of an Applied Geology course meaning that (most of) the polled students were about to acquire a bachelor of science finishing their undergraduate studies. The students were asked multiple questions related to iLectures and their attitude towards this asynchronous content delivery approach as integral part of a flipped classroom. Provided that such a STEM unit with 40-120 students can be deemed representative of the wider student community, the findings indicate that students in general have come to terms with online lectures, way before COVID19 gave them no other choice. Acceptance rates for iLectures were over $50 \%$ across all years, except for 2020, a clear indication that COVID19 marred the online experience, probably due to oversaturation and isolation. The majority of the students saw benefits in this asynchronous lecturing approach, irrespective of whether the rationale behind it had been explained in detail. Despite seeing benefits of the flipped classroom and recorded lectures, one out of three students preferred live lectures. This number has increased after COVID19 to $40 \%$, yet another sign of the negative impact of the pandemic on online lecturing. This inference is unrelated to the quality of the recordings which was deemed high. Finally, the importance of meaningful extended lecture notes to complement the recordings is highlighted.
\end{abstract}

Keywords: Flipped classroom; online lectures; iLectures; COVID19, blended learning. 


\section{Introduction}

Before COVID19, one of the main rationales of desisting from live lecturing and offering online recordings instead (mind, for internal on-campus students) was the flipped classroom approach (FCA). The FCA and its pros and cons has been covered in detail in the literature (Akçayır \& Akçayır, 2018; Bishop \& Verleger, 2013; Milman, 2012; Tucker, 2012) and is per se no the subject of this study. It is understood (and applied) here as freeing up face-toface time and using it for tutorials and laboratories where the recorded lecture material is put into practice via hands-on group as well as individual activities (e.g. case studies), discussions, and presentations. It is in these physical spaces where the students are prepared for the subsequent assessment of learning outcomes. As for the online lectures, these are normal 'off-the-shelf' slide-based recordings, nothing extra-ordinary or quirky whose novel and/or unusual format might have had any skewing effect on the polling outcome (more on their format in the text).

Because an integral part of any FCA is asynchronous content delivery, it is important to assess student acceptance (and adoption) of recorded vs. live lecturing, especially now in pandemic times when there is no real alternative to fully online or at least blended learning. There is an increasing amount of studies that have already looked at a comparison between the two techniques (Cardall, Krupat, \& Ulrich, 2008; Gorissen, van Bruggen, \& Jochems, 2012; Gupta \& Saks, 2013; Milman, 2012; Tamm, Ernst, \& Weems, 1999) but none of them has been carried out over several years. They provide a glimpse from a single cohort over a single term -and vary in their findings whether recorded lectures present a benefit over live ones. To help gauge student disposition towards iLectures before and after COVID19 and whether this attitude changes demographically and (somewhat) generationally over years, the introduction of the FCA was accompanied by rolling out a questionnaire. This ethics approved questionnaire enabled the compilation of empirical data on sundry aspects of recorded lectures that shed light on student behaviour, perception, and expectancy.

\section{Results}

Table 1 displays the ten questions administered to the students in the last week of the semester. They are part of a larger online questionnaire generated with Google Forms. The target group are $3^{\text {rd }}$ year geology (and ecology, bioscience, and water technology) undergraduate students enrolled in a unit called 'Environmental Geoscience', taught during semester 2. This unit is one of the last four units that a prospective Bachelor of Science student takes before graduating. The students are a mix of overseas (15\%) and domestic students ( $85 \%$ ), from different countries and Australian States respectively, and from varying personal backgrounds; traditional as well as non-traditional students, most between 20 and 35 years of age. The considerably varying student numbers (increasing 1/3 from 2015 to 2017 
and then slumping 2/3 until 2020, see Fig.1 caption) are due to the intimate coupling of employment prospects for geologists and the welfare of the mining industry in Western Australia. Figure 1 summarises the students' responses to the first eight questions. Figure 1a displays their response to the most important question, viz. whether they found recorded lectures better than live ones. The adjectives 'better' and 'worse' were not specified, so they may as well qualify as 'more entertaining-engaging' (rather than 'better') as well as less 'educating-informative-interesting' (rather than 'worse'). As can be seen, around a third of the students could not make up their minds (\#3) while a similar percentage was favourable (\#4). And those finding iLectures far better (\#5) clearly outnumbered those who considered them worse (\#2) or far worse (\#1). What is striking, though, is that the student cohort who deemed iLectures better in 2019 (\#4) precipitously dropped from 45 to $25 \%$ in 2020 while at the same time the numbers of undecided students (\#3) increased from 27 to $47 \%$. It is hard not to blame COVID19 for this patent trend against online lecturing. While the content of this particular unit was fine-tuned to online delivery over the years, in semester 1 of 2020 (an academic year in Australia follows a calendar year), other $3^{\text {rd }}$ year units (and field excursions) had to change and adapt to a fully online environment practically overnight and this 'rush' may explain this huge decrease in favourable judgement from 2019 to 2020. It appears as though an increasing number of students have become disenchanted with iLectures as way of content delivery and less sure about their benefits.

Table 1. The full questions related to iLecture acceptance.

Did you find the iLecture format better than live lectures?

In this unit the stipulated time for the iLectures was max. $2 \mathrm{~h}$ which I usually separated into shorter sound bites, sometimes less than $1 \mathrm{~h}$ total. Do you think that time frame is reasonable?

What is the number of iLectures that you listened to?

And for each iLecture, what was the percentage of completion? Did you listen to it all or did you skim through it?

How important are the lecture notes, to what degree do you rely on them?

I provided Blenspace links to focus in far more detail on certain concepts that I cannot cover in the iLecture. Did you take advantage of this offer?

Would you like to see more YouTube videos to illustrate certain concepts?

I am considering providing only lecture notes and YouTube and Blendspace links to convey the lecture material, rather than recorded lectures. The rationale behind is that lecturers are more curators than generators of information. What is your take on such an approach? Yes (I can imagine that this approach works fine because I primarily rely on the lecture notes and feel comfortable to complement that information with short YouTube videos rather than entire iLectures); Undecided (Sometimes the content is better presented by the lecturer, sometimes notes and YouTube links simply do); No (I want to listen to the lecturer)

What about the learning effect from the iLectures, was there any in your opinion?

*Do you agree with the alleged benefits of online lecturing or are you critical and still favour live lectures?

*Question added after 2016 


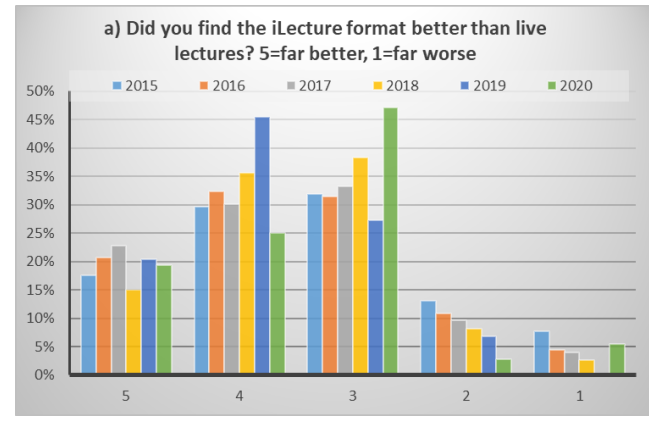

c) What is the number ( $n=12)$ of ilectures that you listened to?

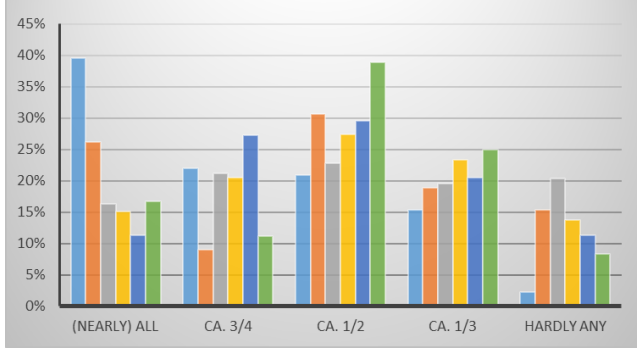

e) How important are the lecture notes, to what degree do you rely on them? $5=$ absolutely vital, $1=$ not at all

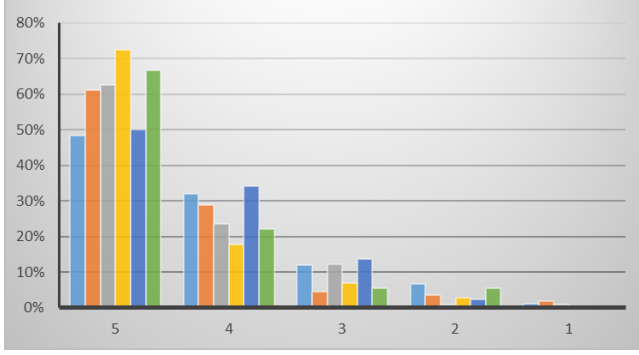

g) Would you like to see more YouTube videos?

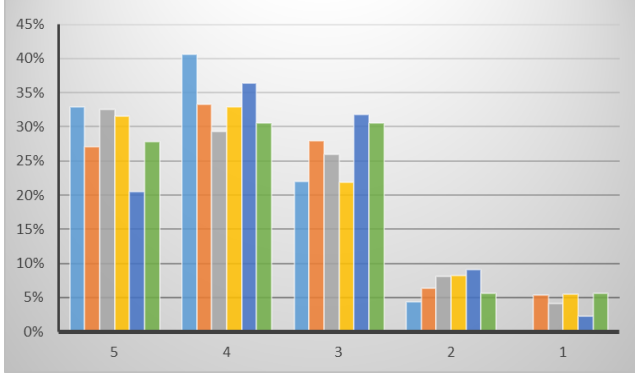

b) Do you think $2 \mathrm{~h}$ iLectures are reasonable?

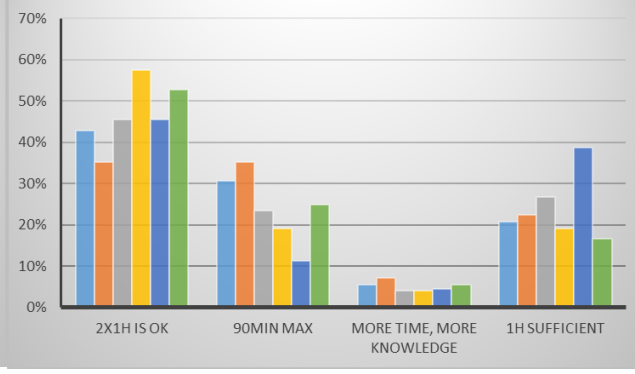

d) What was the percentage of completion?

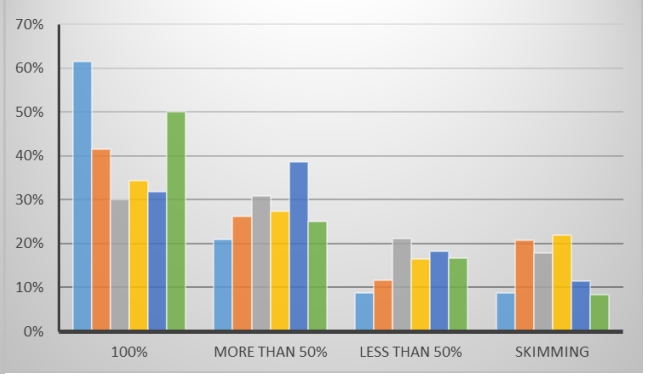

f) Did you take advantage of the Blenspace links?

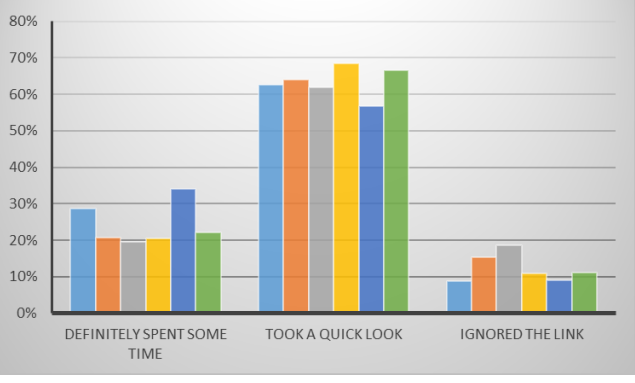

h) Lecture notes, YouTube and Blendspace links or rather recorded lectures?

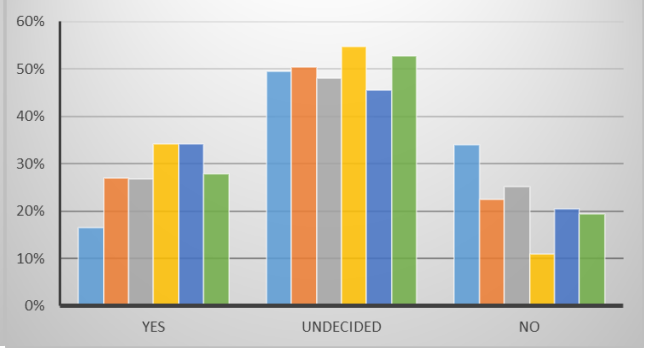

Figure 1. Eight questions and their respective student responses (a-h) to a questionnaire implemented over six years from 2015 to 2020, see legend in (a). The full questions have been tabulated in Table 1. The polling numbers for each year are as follows: $n=91$ (2015), $n=111$ (2016), $n=123$ (2017), $n=73$ (2018), $n=44$ (2019), $n=36$ (2020). 
It is legitimate to ask if this change of mind is related to the iLecture format or quality or a change in the overall student behaviour for which the next graphs will provide information. Figure $1 \mathrm{~b}$ displays student answers related to the length of recording, usually an important feature for acceptance. Mind that while the official tuition pattern called for lectures over two hours, none of the individual recordings were longer than 40 to $60 \mathrm{~min}$. What surprises is that at least $40 \%$ of the students do not find issue with a (ludicrously long) stipulated recording over two hours, irrespective of the year sampled. More perplexing is that this percentage went even over $50 \%$ in 2020 (and sky-rocketed in 2018 to $58 \%$ ). Not surprisingly, there is a portion of the bulk thinking that one hour is more than sufficient but except for 2019, this fraction was around 20-25\%, well below the other end of the spectrum. It would, however, be premature to shout victory given these encouraging numbers. After all, Figure 1c demonstrates that the numbers of lectures the students listened to varied considerably across the years. From the twelve recordings (a regular teaching semester consists of twelve tuition weeks in Australia), many if not all lectures were listened to primarily in 2015 and 2019 while in 2017,2018 , and 2020 the sobering truth is that around $40 \%$ of the students listened to hardly any or only around a third of the recordings. Equally revealing is the number of lectures that the students listened to completion (Fig. 1d). Only in 2015 did the majority of students (62\%) listen to completion whereas this number was between 30 and $40 \%$ from 2016 to 2019 . Roughly a third of the cohort did only skim through or digested less than 50 $\%$ of the recordings, irrespective of the year polled (except for the 'diligent' year 2015). As a sideline, many electronic learning management systems nowadays allow tracking and data collection of iLecture consumption to garner more accurate quantitative information to avoid such personal perceptions as 'nearly all' or 'skimming' but they only work when the online content is streamed, i.e. under constant internet connection. This was not feasible as basically all students insisted on the option of downloading recordings that enabled them to watch at their leisure, pace, and independent of the internet connection and location. The percentages from Figure 1c and 1d explain why students did not have a problem with the length of the recording. It seems they adjusted their weekly content intake by cutting down on the number of videos they watched and the time they spent on an iLecture. This assumption is corroborated by Figure 1e that displays the reliance on lecture notes. Across the board, the (vast) majority of students found the extensive notes either vital (\#4) or absolutely vital (\#5) and primarily relied on them rather than the spoken word. These notes (one student called them 'beautifully crafted') are not merely PowerPoint handouts but narratives in themselves in order to enhance rather than reproduce the lecture content; the latter has been shown not to aid on test performances (Noppe, Achterberg, Duquaine, Huebbe, \& Williams, 2007) or in deeper learning (Kinchin, 2006). It is noteworthy that this utter reliance (\#5) on lecture notes fluctuates over the years and cohort, ranging from $48 \%$ (2015) to $73 \%$ (2018). This scatter notwithstanding, it is patent that only a marginal fraction of students displayed (utter) indifference to this auxiliary medium. Speaking of which, the iLectures included links to 
curated Blendspaces and YouTube videos to liven up the presentation and provide alternative ways of content delivery. Blendspaces are multimedia digital lessons finding their way into mainstream online teaching to enhance the self-directed flipped classroom experience (Devaki \& Deivam, 2017; Zainuddin \& Perera, 2018). The feedback to their implementation in this unit was tepid, at best. Over the years, 20 to $30 \%$ of the students took up this offer (Fig. 1f) but a fairly homogeneous $60 \%$ of the students had merely a peek, not really enough for any didactic effect to take hold. As for the offer of introducing more YouTube links, the bulk of students were favourable to this idea (\#5 and 4 in Fig. 1g) but given the selective approach of what and for how long students are watching, it remains doubtful if more YouTube videos can win the day and displace recorded lectures as more favoured medium. To find the students' predilection, they were asked if they could imagine themselves relying solely on a mix of notes, Blendspaces, and YouTube videos for content delivery rather than recorded lectures (Fig. 1h). The answer was that it all depends ('undecided', see full text for meaning in Table 1). The student desired (not surprisingly) the best of all worlds which means notes and short Blendspace/Youtube gigs in some instances and entire two hour long recordings at other times. So far these observations have not considered the quality of the recording; after all no human can stomach a dry, droning-on recording of two hours or irrelevant or non-pertinent multi-media links. The easiest, albeit not necessarily most scientific, way to gauge the quality of the iLectures was to ask the students straight whether they thought they had actually learned something from them. This is clearly a subjective notion but unavoidable in any observational study involving student perception. Figure 2a illustrates that the majority of students across the years were indeed under the impression of having learned a lot while $40 \%$ of them thought they had learned at least something. Only a negligible fraction below $5 \%$ was not gaining anything (worthwhile) from them. It seems perplexing that, despite the apparent quality and variety of the digital multi-media material, the motivation to engage with it was lacking (see Fig. 1c, d). A possible explanation can be found in a study where students used recorded lectures primarily as a replacement for missed lectures or study tasks (preparing for an assessment) whereas the quality of the actual lectures appeared not to influence the use of the recorded lectures (Gorissen et al., 2012). 


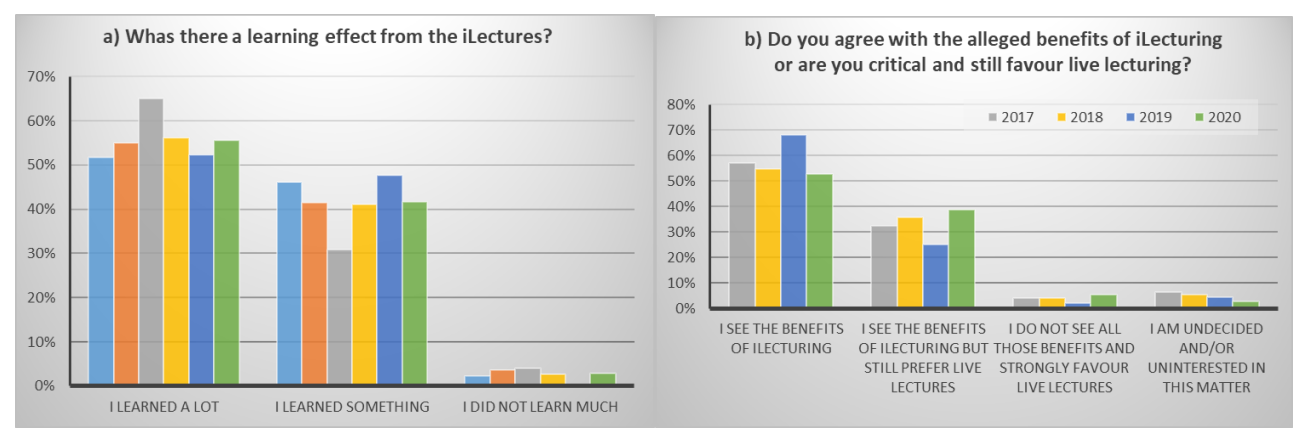

Figure 2. Two more questions and their respective student responses from the questionnaire.

If quality appears secondary in importance, what other intrinsic factors can prompt the students to confront the unit content? FCA relies heavily on self-directed learning and therefore the student needs to understand the rationale behind this switch from live to online lecturing. To foster such understanding, starting in 2017 the benefits of FCA (e.g. self-paced learning, pause button, maximum flexibility, no time constraints, class time dedicated to hands-on face-to-face activities etc.) were explicitly spelled out in a separate pdf file at the start of the unit. As can be seen in Figure 1a, this pro-active approach did not make a dent in approval rates before and after 2017. Also from 2017 onwards, the students were asked more specifically if they saw the benefits of iLecturing (as integral part of FCA). Depsite the observation that the majority clearly saw the benefits, a pernicious roughly $30 \%$ of the cohort has, continuously over the years, still preferred live lectures (see Fig. 2b). How come students are aware of certain benefits but choose a more personal approach, even if that 'tentatively' means less learning? Admittedly, whether live or digital content delivery is a more efficient didactic tool remains undecided; Bahnson and Olejnikova (2017) for example found that student learning was not improved by substituting a self-paced, recorded module for live instruction. This said, the students of this unit are most likely not aware of the ongoing discussion and have been primed to think in positive terms about iLectures and FCA. Still, the desire for a physical experience trumps any touted FCA benefits. This sounds understandable but attendance rates of the same cohort in associated $3^{\text {rd }}$ year units whose lectures were live (before 2020) consistently remained $\leq 20 \%$. So while a certain portion of the students claimed preference for live sessions, not all would de facto attend them. By the way, this preference for live lectures (Fig. 2b) jumped considerably from $25 \%$ in 2019 to 39 $\%$ in 2020 while support for iLectures declined from 68 to $53 \%$ in the same interval; an indication that COVID19 left the students saturated with digital learning. Fortunately, the fraction of students who were either indifferent to the entire issue or just did not see any advantages in asynchronous content delivery was consistently $\leq 7 \%$ in the past four years. 


\section{Conclusions}

Inasmuch as this STEM unit can be deemed reasonably representative of a wider student attitude on or rather off campus, at least at Australian universities, the following more general conclusions can be drawn:

- Most graphs show scatter, i.e. varied annual student responses/attitudes. There is no apparent trend for any particular question across the years (going consistently up or down) to suggest any trend or development towards one particular leaning. In the few instances where the annual answers are comparable (Fig. 1f, h), that may be an artefact because there were only three options with the middle ground taking up most votes.

- A couple of graphs show clear signs of student weariness or saturation vis-à-vis online lecturing from 2019 to 2020, i.e. after COVID19 struck (Figs. 1a, 2b). Before that year, however, acceptance rates for online delivery of unit content were over $50 \%$ (Fig. 1a, sum of \#4 and \#5), meaning that the bulk of students accepted iLectures as viable means of content delivery.

- Despite efforts to 'sell' the pedagogic advantages of online lectures as part of the FCA, roughly a third of the student bulk remained steadfast in their support for synchronous content delivery (Fig. 2b), especially but not exclusively in 2020. Face to face time is clearly appreciated and sought, more so during the period of isolation.

- Students managed the time spend on an iLecture, irrespective of the duration of the recording, which varied between 40 and $60 \mathrm{~min}$. The students decided which iLectures and for how long they listened to. This discretion is likely a direct consequence of providing extended notes that enabled the students to rely solely on the written text to prepare adequately for the practical tutorials and laboratories.

\section{References}

Akçayır, G., \& Akçayır, M. (2018). The flipped classroom: A review of its advantages and challenges. Computers \& Education, 126, 334-345.

Bahnson, J., \& Olejnikova, L. (2017). Are Recorded Lectures Better than Live Lectures for Teaching Students Legal Research. Law Libr. J., 109, 187.

Bishop, J. L., \& Verleger, M. A. (2013). The flipped classroom: A survey of the research. Paper presented at the ASEE national conference proceedings, Atlanta, GA.

Cardall, S., Krupat, E., \& Ulrich, M. (2008). Live Lecture Versus Video-Recorded Lecture: Are Students Voting With Their Feet? Academic Medicine, 83(12).

Devaki, N., \& Deivam, M. (2017). Synergising Blendspace for Effective Instruction. International Journal of Computer Science Trends and Technology, 5(2), 4.

Gorissen, P., van Bruggen, J., \& Jochems, W. (2012). Students and recorded lectures: survey on current use and demands for higher education. Research in Learning Technology, 20(0). doi:10.3402/rlt.v20i0.17299 
Gupta, A., \& Saks, N. S. (2013). Exploring medical student decisions regarding attending live lectures and using recorded lectures. Medical Teacher, 35(9), 767-771.

Kinchin, I. M. (2006). Developing PowerPoint handouts to support meaningful learning. BJET, 37(4), 647-650.

Milman, N. B. (2012). The flipped classroom strategy: What is it and how can it best be used? Distance learning, 9(3), 85.

Noppe, I. C., Achterberg, J., Duquaine, L., Huebbe, M., \& Williams, C. (2007). PowerPoint Presentation Handouts and College Student Learning Outcomes. International Journal for the Scholarship of Teaching and Learning, 1(1), 9.

Tamm, E. P., Ernst, R., \& Weems, W. (1999). The virtual lecture: delivery of live and recorded presentations over the Internet. AJR. American journal of roentgenology, 172(1), 9-12.

Tucker, B. (2012). The flipped classroom. Education next, 12(1), 82-83.

Zainuddin, Z., \& Perera, C. J. (2018). Supporting students' self-directed learning in the flipped classroom through the LMS TES BlendSpace. On the Horizon. 